\title{
Fusion Procedure for Cyclotomic Hecke Algebras
}

Oleg V. OGIEVETSKY $Y^{\dagger^{1} \dagger^{2} \dagger^{3}}$ and Lö̈c POULAIN D'ANDECY ${ }^{\dagger^{4}}$

$\dagger^{1}$ Center of Theoretical Physics, Aix Marseille Université, CNRS, UMR 7332, 13288 Marseille, France

E-mail: oleg@cpt.univ-mrs.fr

$\dagger^{2}$ Université de Toulon, CNRS, UMR 7332, 83957 La Garde, France

$\dagger^{3}$ On leave of absence from P.N. Lebedev Physical Institute, Leninsky Pr. 53, 117924 Moscow, Russia

$\dagger^{4}$ Mathematics Laboratory of Versailles, LMV, CNRS UMR 8100, Versailles Saint-Quentin University, 45 avenue des Etas-Unis, 78035 Versailles Cedex, France

E-mail: L.B.PoulainDAndecy@uva.nl

Received September 28, 2013, in final form March 29, 2014; Published online April 01, 2014 http://dx.doi.org/10.3842/SIGMA.2014.039

\begin{abstract}
A complete system of primitive pairwise orthogonal idempotents for cyclotomic Hecke algebras is constructed by consecutive evaluations of a rational function in several variables on quantum contents of multi-tableaux. This function is a product of two terms, one of which depends only on the shape of the multi-tableau and is proportional to the inverse of the corresponding Schur element.
\end{abstract}

Key words: cyclotomic Hecke algebras; fusion formula; idempotents; Young tableaux; JucysMurphy elements; Schur element

2010 Mathematics Subject Classification: 20C08; 05E10

\section{Introduction}

This article is a continuation of the article [14] on the fusion procedure for the complex reflection groups $G(m, 1, n)$. The cyclotomic Hecke algebra $H(m, 1, n)$, introduced in $[2,3,4]$, is a natural flat deformation of the group ring of the complex reflection group $G(m, 1, n)$.

In [14], a fusion procedure, in the spirit of [12], for the complex reflection groups $G(m, 1, n)$ is suggested: a complete system of primitive pairwise orthogonal idempotents for the groups $G(m, 1, n)$ is obtained by consecutive evaluations of a rational function in several variables with values in the group ring $\mathbb{C} G(m, 1, n)$. This approach to the fusion procedure relies on the existence of a maximal commutative set of elements of $\mathbb{C} G(m, 1, n)$ formed by the Jucys-Murphy elements.

Jucys-Murphy elements for the cyclotomic Hecke algebra $H(m, 1, n)$ were introduced in [2] and were used in [13] to develop an inductive approach to the representation theory of the chain of the algebras $H(m, 1, n)$. In the generic setting or under certain restrictions on the parameters of the algebra $H(m, 1, n)$ (see Section 2 for precise definitions), the Jucys-Murphy elements form a maximal commutative set in the algebra $H(m, 1, n)$.

A complete system of primitive pairwise orthogonal idempotents of the algebra $H(m, 1, n)$ is indexed by the set of standard $m$-tableaux of size $n$. We formulate here the main result of the article. Let $\boldsymbol{\lambda}$ be an $m$-partition of size $n$ and $\mathcal{T}$ be a standard $m$-tableau of shape $\boldsymbol{\lambda}$.

${ }^{\star}$ This paper is a contribution to the Special Issue in honor of Anatol Kirillov and Tetsuji Miwa. The full collection is available at http://www.emis.de/journals/SIGMA/InfiniteAnalysis2013.html 
Theorem. The idempotent $E_{\mathcal{T}}$ of $H(m, 1, n)$ corresponding to the standard $m$-tableau $\mathcal{T}$ of shape $\boldsymbol{\lambda}$ can be obtained by the following consecutive evaluations

$$
E_{\mathcal{T}}=\left.\left.\left.\mathrm{F}_{\lambda} \Phi\left(u_{1}, \ldots, u_{n}\right)\right|_{u_{1}=c_{1}} \cdots\right|_{u_{n-1}=c_{n-1}}\right|_{u_{n}=c_{n}}
$$

Here $\Phi\left(u_{1}, \ldots, u_{n}\right)$ is a rational function with values in the algebra $H(m, 1, n), \mathbf{F}_{\boldsymbol{\lambda}}$ is an element of the base ring and $c_{1}, \ldots, c_{n}$ are the quantum contents of the $m$-nodes of $\mathcal{T}$.

The classical limit of our fusion procedure for algebras $H(m, 1, n)$ reproduces the fusion procedure of [14] for the complex reflection groups $G(m, 1, n)$. For $\mathbb{C} G(m, 1, n)$, the variables of the rational function are split into two parts, one is related to the position of the $m$-node (its place in the $m$-tuple) and the other one - to the classical content of the $m$-node. The position variables can be evaluated simultaneously while the classical content variables have then to be evaluated consequently from 1 to $n$. For the algebra $H(m, 1, n)$, the information about positions and classical contents is fully contained in the quantum contents, and now the function $\Phi$ depends on only one set of variables.

Remarkably, the coefficient $F_{\boldsymbol{\lambda}}$ appearing in (1) depends only on the shape $\boldsymbol{\lambda}$ of the standard $m$-tableau $\mathcal{T}$ (cf. with the more delicate fusion procedure for the Birman-Murakami-Wenzl algebra [7]). In the classical limit, this coefficient depends only on the usual hook length, see [14]. However, in the deformed situation, the calculation of $F_{\boldsymbol{\lambda}}$ needs a non-trivial generalization of the hook length. It appears that the coefficient $\mathrm{F}_{\boldsymbol{\lambda}}$ is proportional to the inverse of the Schur element (corresponding to the $m$-partition $\boldsymbol{\lambda}$ ) associated to a specific symmetrizing form on the algebra $H(m, 1, n)$ (see $[6,11]$ for a calculation of these Schur elements and [5] for an expression in terms of generalized hook lengths); for more precise statements, we refer to [15] where we calculate, using the fusion formula presented here, weights of certain central forms and in particular of these Schur elements.

For $m=1$, the cyclotomic Hecke algebra $H(1,1, n)$ is the Hecke algebra of type $\mathrm{A}$ and our fusion procedure reduces to the fusion procedure for the Hecke algebra in [8]. The factors in the rational function are arranged in [8] in such a way that there is a product of "Baxterized" generators on one side and a product of non-Baxterized generators on the other side. For $m>1$ a rearrangement, as for the type $\mathrm{A}$, of the rational function appearing in (1) is no more possible.

The additional, with respect to $H(1,1, n)$, generator of $H(m, 1, n)$ satisfies the reflection equation whose "Baxterization" is known [9]. But - and this is maybe surprising - the full Baxterized form is not used in the construction of the rational function in (1). The rational expression involving the additional generator satisfies only a certain limit of the reflection equation with spectral parameters.

The Hecke algebra of type A is the natural quotient of the Birman-Murakami-Wenzl algebra. The fusion procedure, developed in [7], for the Birman-Murakami-Wenzl algebra provides a oneparameter family of fusion procedures for the Hecke algebra of type A. We think that for $m>1$ the fusion procedure (1) can be included into a one-parameter family as well.

\section{Definitions}

\subsection{Cyclotomic Hecke algebra and Baxterized elements}

Let $m \in \mathbb{Z}_{>0}$ and $n \in \mathbb{Z}_{\geq 0}$. Let $q, v_{1}, \ldots, v_{m}$ be complex numbers with $q \neq 0$. The cyclotomic Hecke algebra $H(m, 1, n+1)$ is the unital associative algebra over $\mathbb{C}$ generated by $\tau, \sigma_{1}, \ldots, \sigma_{n}$ with the defining relations

$$
\begin{array}{ll}
\sigma_{i} \sigma_{i+1} \sigma_{i}=\sigma_{i+1} \sigma_{i} \sigma_{i+1} & \text { for } i=1 \ldots, n-1, \\
\sigma_{i} \sigma_{j}=\sigma_{j} \sigma_{i} & \text { for } i, j=1, \ldots, n \text { such that }|i-j|>1,
\end{array}
$$




$$
\begin{array}{ll}
\tau \sigma_{1} \tau \sigma_{1}=\sigma_{1} \tau \sigma_{1} \tau, & \\
\tau \sigma_{i}=\sigma_{i} \tau & \text { for } i>1, \\
\sigma_{i}^{2}=\left(q-q^{-1}\right) \sigma_{i}+1 & \text { for } i=1, \ldots, n, \\
\left(\tau-v_{1}\right) \cdots\left(\tau-v_{m}\right)=0 . &
\end{array}
$$

We define $H(m, 1,0):=\mathbb{C}$. The cyclotomic Hecke algebras $H(m, 1, n)$ form a chain (with respect to $n$ ) of algebras defined by inclusions $H(m, 1, n) \ni \tau, \sigma_{1}, \ldots, \sigma_{n-1} \mapsto \tau, \sigma_{1}, \ldots, \sigma_{n-1} \in$ $H(m, 1, n+1)$ for any $n \geq 0$. These inclusions allow to consider (as it will often be done in the article) elements of $H(m, 1, n)$ as elements of $H\left(m, 1, n+n^{\prime}\right)$ for any $n^{\prime}=0,1,2, \ldots$

In the sequel we assume the following restrictions on the parameters $q, v_{1}, \ldots, v_{m}$ :

$$
\begin{aligned}
& 1+q^{2}+\cdots+q^{2 N} \neq 0 \text { for } N \text { such that } N \leq n, \\
& q^{2 i} v_{j}-v_{k} \neq 0 \text { for } i, j, k \text { such that } j \neq k \text { and }-n \leq i \leq n, \\
& v_{j} \neq 0 \text { for } j=1, \ldots, m .
\end{aligned}
$$

The restrictions (2), (3) are necessary and sufficient for the semi-simplicity of the algebra $H(m, 1, n+1)$ [1, main theorem]. The restriction (4) is necessary for the maximality of the commutative set of the Jucys-Murphy elements (as defined in Section 3) [1, Proposition 3.2].

Define the following rational functions in variables $a, b$ with values in $H(m, 1, n+1)$ :

$$
\bar{\sigma}_{i}(a, b):=\sigma_{i}+\left(q-q^{-1}\right) \frac{b}{a-b}, \quad i=1, \ldots, n .
$$

The functions $\bar{\sigma}_{i}$ are called Baxterized elements and the variables $a$ and $b$ are called spectral parameters. These Baxterized elements satisfy the Yang-Baxter equation with spectral parameters

$$
\bar{\sigma}_{i}(a, b) \bar{\sigma}_{i+1}(a, c) \bar{\sigma}_{i}(b, c)=\bar{\sigma}_{i+1}(b, c) \bar{\sigma}_{i}(a, c) \bar{\sigma}_{i+1}(a, b) .
$$

The following formula will be used later

$$
\bar{\sigma}_{i}(a, b) \bar{\sigma}_{i}(b, a)=\frac{\left(a-q^{2} b\right)\left(a-q^{-2} b\right)}{(a-b)^{2}} \quad \text { for } \quad i=1, \ldots, n .
$$

Let $\mathfrak{p}_{i}, i=1, \ldots, m$, be the eigen-idempotents of $\tau, \mathfrak{p}_{i}:=\prod_{j: j \neq i}\left(\tau-v_{j}\right) /\left(v_{i}-v_{j}\right)$, so that $\tau \mathfrak{p}_{i}=v_{i} \mathfrak{p}_{i}, \mathfrak{p}_{i} \mathfrak{p}_{j}=\delta_{i j} \mathfrak{p}_{i}, \sum_{i} \mathfrak{p}_{i}=1$ and $\tau=\sum_{i} v_{i} \mathfrak{p}_{i}$. Let $r$ be an indeterminate. The resolvent $(r-\tau)^{-1}:=\sum_{i}\left(r-v_{i}\right)^{-1} \mathfrak{p}_{i}$ of $\tau$ is an element of $\mathbb{C}(r) \otimes_{\mathbb{C}} H(m, 1, n+1)$. Define a rational function $\bar{\tau}$ with values in $H(m, 1, n+1)$ :

$$
\bar{\tau}(r):=\frac{\left(r-v_{1}\right)\left(r-v_{2}\right) \cdots\left(r-v_{m}\right)}{r-\tau}=\sum_{i}\left(\prod_{j: j \neq i}\left(r-v_{j}\right)\right) \mathfrak{p}_{i} \in \mathbb{C}[r] \otimes_{\mathbb{C}} H(m, 1, n+1) .
$$

Remarks. (i) The function $\bar{\tau}(r)$ can be expressed in terms of the complex numbers $a_{0}, a_{1}, \ldots, a_{m}$ defined by

$$
\left(X-v_{1}\right)\left(X-v_{2}\right) \cdots\left(X-v_{m}\right)=a_{0}+a_{1} X+\cdots+a_{m} X^{m}
$$

where $X$ is an indeterminate. Let $\mathfrak{a}_{i}(r), i=0, \ldots, m$, be the polynomials in $r$ given by

$$
\mathfrak{a}_{i}(r)=a_{i}+r a_{i+1}+\cdots+r^{m-i} a_{m} \quad \text { for } \quad i=0, \ldots, m \text {. }
$$


Using that $r \mathfrak{a}_{i+1}(r)=\mathfrak{a}_{i}(r)-a_{i}$, for $i=0, \ldots, m-1$, it is straightforward to verify that

$$
(r-\tau) \sum_{i=0}^{m-1} \mathfrak{a}_{i+1}(r) \tau^{i}=\mathfrak{a}_{0}(r)=\left(r-v_{1}\right)\left(r-v_{2}\right) \cdots\left(r-v_{m}\right) .
$$

It follows from (9) that

$$
\bar{\tau}(r)=\mathfrak{a}_{1}(r)+\mathfrak{a}_{2}(r) \tau+\cdots+\mathfrak{a}_{m}(r) \tau^{m-1}=\sum_{i=0}^{m-1} \mathfrak{a}_{i+1}(r) \tau^{i},
$$

For example, for $m=1$, we have $\bar{\tau}(r)=1$; for $m=2$, we have $\bar{\tau}(r)=\tau+r-v_{1}-v_{2}$; for $m=3$, we have $\bar{\tau}(r)=\tau^{2}+\left(r-v_{1}-v_{2}-v_{3}\right) \tau+r^{2}-r\left(v_{1}+v_{2}+v_{3}\right)+v_{1} v_{2}+v_{1} v_{3}+v_{2} v_{3}$.

(ii) The functions $\bar{\tau}$ and $\bar{\sigma}_{1}$ satisfy the following equation

$$
\bar{\sigma}_{1}(a, b) \bar{\tau}(a) \sigma_{1}^{-1} \bar{\tau}(b)=\bar{\tau}(b) \sigma_{1}^{-1} \bar{\tau}(a) \bar{\sigma}_{1}(a, b) .
$$

Indeed, due to (6) and (7), the equality (11) is equivalent to

$$
(\tau-b) \sigma_{1}(\tau-a) \bar{\sigma}_{1}(b, a)=\bar{\sigma}_{1}(b, a)(\tau-a) \sigma_{1}(\tau-b),
$$

which is proved by a straightforward calculation. The equation (11) is a certain (we leave the details to the reader) limit of the usual reflection equation with spectral parameters (see, for example, [10]).

\section{$2.2 m$-partitions, m-tableaux and generalized hook length}

Let $\lambda \vdash n+1$ be a partition of size $n+1$, that is, $\lambda=\left(\lambda_{1}, \ldots, \lambda_{l}\right)$, where $\lambda_{j}, j=1, \ldots, l$, are positive integers, $\lambda_{1} \geqslant \lambda_{2} \geqslant \cdots \geqslant \lambda_{l}$ and $n+1=\lambda_{1}+\cdots+\lambda_{l}$. We identify partitions with their Young diagrams: the Young diagram of $\lambda$ is a left-justified array of rows of nodes containing $\lambda_{j}$ nodes in the $j$-th row, $j=1, \ldots, l$; the rows are numbered from top to bottom. For a node $\alpha$ in line $x$ and column $y$ of a Young diagram, we denote $\alpha=(x, y)$ and call $x$ and $y$ the coordinates of the node.

An $m$-partition, or a Young $m$-diagram, of size $n+1$ is an $m$-tuple of partitions such that the sum of their sizes equals $n+1$; e.g. the Young 3-diagram $(\square \square, \square, \square)$ represents the 3-partition $((2),(1),(1))$ of size 4 .

We shall understand an $m$-partition as a set of $m$-nodes, where an $m$-node $\boldsymbol{\alpha}$ is a pair $\{\alpha, k\}$ consisting of a node $\alpha$ and an integer $k=1, \ldots, m$, indicating to which diagram in the $m$-tuple the node belongs. The integer $k$ will be called position of the $m$-node, and we set $\operatorname{pos}(\boldsymbol{\alpha}):=k$.

For an $m$-partition $\boldsymbol{\lambda}$, an $m$-node $\boldsymbol{\alpha}$ of $\boldsymbol{\lambda}$ is called removable if the set of $m$-nodes obtained from $\boldsymbol{\lambda}$ by removing $\boldsymbol{\alpha}$ is still an $m$-partition. An $m$-node $\boldsymbol{\beta}$ not in $\boldsymbol{\lambda}$ is called addable if the set of $m$-nodes obtained from $\boldsymbol{\lambda}$ by adding $\boldsymbol{\beta}$ is still an $m$-partition. For an $m$-partition $\boldsymbol{\lambda}$, we denote by $\mathcal{E}_{-}(\boldsymbol{\lambda})$ the set of removable $m$-nodes of $\boldsymbol{\lambda}$ and by $\mathcal{E}_{+}(\boldsymbol{\lambda})$ the set of addable $m$-nodes of $\boldsymbol{\lambda}$. For example, the removable/addable $m$-nodes (marked with $-/+$ ) for the 3-partition $(\square \square, \square, \square)$ are

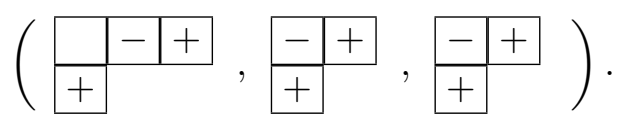

Let $\boldsymbol{\lambda}$ be an $m$-diagram of size $n+1$. A standard $m$-tableau of shape $\boldsymbol{\lambda}$ is obtained by placing the numbers $1, \ldots, n+1$ in the $m$-nodes of the diagrams of $\boldsymbol{\lambda}$ in such a way that the numbers in the nodes ascend along rows and down columns in every diagram. The size of a standard $m$-tableau is the size of its shape. 
Let $q, v_{1}, \ldots, v_{m}$ be the parameters of the cyclotomic Hecke algebra $H(m, 1, n+1)$ and let $\boldsymbol{\alpha}=\{\alpha, k\}$ be an $m$-node with $\alpha=(x, y)$. We denote by $c c(\boldsymbol{\alpha})$ the classical content of the node $\alpha$, $c c(\boldsymbol{\alpha}):=y-x$, and by $c(\boldsymbol{\alpha})$ the quantum content of the $m$-node $\boldsymbol{\alpha}, c(\boldsymbol{\alpha}):=v_{k} q^{2 c c(\boldsymbol{\alpha})}=v_{k} q^{2(y-x)}$.

For a standard $m$-tableau $\mathcal{T}$ of shape $\boldsymbol{\lambda}$ let $\boldsymbol{\alpha}_{i}$ be the $m$-node of $\mathcal{T}$ occupied by the number $i$, $i=1, \ldots, n+1 ;$ we set $c(\mathcal{T} \mid i):=c\left(\boldsymbol{\alpha}_{i}\right), c c(\mathcal{T} \mid i):=c c\left(\boldsymbol{\alpha}_{i}\right)$ and $\operatorname{pos}(\mathcal{T} \mid i):=\operatorname{pos}\left(\boldsymbol{\alpha}_{i}\right)$. For example,

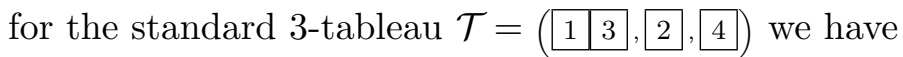

$$
\begin{array}{lllll}
c(\mathcal{T} \mid 1)=v_{1}, & c(\mathcal{T} \mid 2)=v_{2}, & c(\mathcal{T} \mid 3)=v_{1} q^{2} & \text { and } & c(\mathcal{T} \mid 4)=v_{3}, \\
c c(\mathcal{T} \mid 1)=0, & c c(\mathcal{T} \mid 2)=0, & c c(\mathcal{T} \mid 3)=1 & \text { and } & c c(\mathcal{T} \mid 4)=0, \\
\operatorname{pos}(\mathcal{T} \mid 1)=1, & \operatorname{pos}(\mathcal{T} \mid 2)=2, & \operatorname{pos}(\mathcal{T} \mid 3)=1 & \text { and } & \operatorname{pos}(\mathcal{T} \mid 4)=3,
\end{array}
$$

Generalized hook length. The hook of a node $\alpha$ of a partition $\lambda$ is the set of nodes of $\lambda$ consisting of the node $\alpha$ and the nodes which lie either under $\alpha$ in the same column or to the right of $\alpha$ in the same row; the hook length $h_{\lambda}(\alpha)$ of $\alpha$ is the cardinality of the hook of $\alpha$. We extend this definition to $m$-nodes. For an $m$-node $\boldsymbol{\alpha}=\{\alpha, k\}$ of an $m$-partition $\boldsymbol{\lambda}$, the hook length of $\boldsymbol{\alpha}$ in $\boldsymbol{\lambda}$, which we denote by $h_{\boldsymbol{\lambda}}(\boldsymbol{\alpha})$, is the hook length of the node $\alpha$ in the $k$-th partition of $\boldsymbol{\lambda}$.

Let $\boldsymbol{\lambda}$ be an $m$-partition. For $j=1, \ldots, m$, let $\mathfrak{l}_{\boldsymbol{\lambda}, x, j}$ be the number of nodes in the line $x$ of the $j$-th diagram of $\boldsymbol{\lambda}$, and $\mathfrak{c}_{\boldsymbol{\lambda}, y, j}$ be the number of nodes in the column $y$ of the $j$-th diagram of $\boldsymbol{\lambda}$. The hook length of an $m$-node $\boldsymbol{\alpha}=\{(x, y), k\}$ of $\boldsymbol{\lambda}$ can be rewritten as

$$
h_{\boldsymbol{\lambda}}(\boldsymbol{\alpha})=\mathfrak{l}_{\boldsymbol{\lambda}, x, k}+\mathfrak{c}_{\boldsymbol{\lambda}, y, k}-x-y+1 \text {. }
$$

Define the generalized hook length of $\boldsymbol{\alpha}$ (see also [5]) by

$$
h_{\boldsymbol{\lambda}}^{(j)}(\boldsymbol{\alpha}):=\mathfrak{l}_{\boldsymbol{\lambda}, x, j}+\mathfrak{c}_{\boldsymbol{\lambda}, y, k}-x-y+1 \quad \text { for } \quad j=1, \ldots, m
$$

in particular, $h_{\boldsymbol{\lambda}}^{(k)}(\boldsymbol{\alpha})=h_{\boldsymbol{\lambda}}(\boldsymbol{\alpha})$ is the usual hook length.

For an $m$-partition $\boldsymbol{\lambda}$, we define

$$
\mathrm{F}_{\boldsymbol{\lambda}}=\prod_{\boldsymbol{\alpha} \in \boldsymbol{\lambda}}\left(\frac{q^{c c(\boldsymbol{\alpha})}}{\left[h_{\boldsymbol{\lambda}}(\boldsymbol{\alpha})\right]_{q}} \prod_{\substack{k=1, \ldots, m \\ k \neq \operatorname{pos}(\boldsymbol{\alpha})}} \frac{q^{-c c(\boldsymbol{\alpha})}}{v_{\operatorname{pos}(\boldsymbol{\alpha})} q^{-h_{\lambda}^{(k)}(\boldsymbol{\alpha})}-v_{k} q^{h_{\lambda}^{(k)}(\boldsymbol{\alpha})}}\right),
$$

where $[j]_{q}:=q^{j-1}+q^{j-3}+\cdots+q^{-j+1}$ for a non-negative integer $j$. Under the restrictions (2)-(4), the number $\boldsymbol{F}_{\boldsymbol{\lambda}}$ is well defined for any $m$-partition $\boldsymbol{\lambda}$ of size less or equal to $n+1$ since $h_{\boldsymbol{\lambda}}(\boldsymbol{\alpha}) \leq n+1$ and $h_{\boldsymbol{\lambda}}^{(k)}(\boldsymbol{\alpha}) \leq n$ if $k \neq \operatorname{pos}(\boldsymbol{\alpha})$ for any $\boldsymbol{\alpha} \in \boldsymbol{\lambda}$.

\section{Idempotents and Jucys-Murphy elements of $H(m, 1, n+1)$}

In this section we recall the definition and some properties, from [2], of the Jucys-Murphy elements of the algebra $H(m, 1, n+1)$, together with some facts about an explicit realization of the irreducible representations of $H(m, 1, n+1)$. We then derive, in the same spirit as in [12], an inductive formula, that we will use in the next section, for the primitive idempotents corresponding to this realization.

The Jucys-Murphy elements $J_{i}, i=1, \ldots, n+1$, of the algebra $H(m, 1, n+1)$ are defined by the following initial condition and recursion

$$
J_{1}=\tau \quad \text { and } \quad J_{i+1}=\sigma_{i} J_{i} \sigma_{i}, \quad i=1, \ldots, n .
$$


We recall that, under the restrictions (2)-(4), the elements $J_{i}, i=1, \ldots, n+1$, form a maximal commutative set (that is, generate a maximal commutative subalgebra) of $H(m, 1, n+1)$ [2, Proposition 3.17]. Recall also that

$$
J_{i} \sigma_{k}=\sigma_{k} J_{i} \quad \text { for } \quad k \neq i-1, i .
$$

The isomorphism classes of irreducible $\mathbb{C}$-representations of $H(m, 1, n+1)$ are in bijection with the set of $m$-partitions of size $n+1$. We use the labeling and the explicit realization of the irreducible representations of $H(m, 1, n+1)$ given in [2]. Namely, for any $m$-partition $\boldsymbol{\lambda}$ of size $n+1$, the irreducible representation $V_{\boldsymbol{\lambda}}$ of $H(m, 1, n+1)$ corresponding to $\boldsymbol{\lambda}$ has a basis $\left\{v_{\mathcal{T}}\right\}$ indexed by the set of standard $m$-tableaux of shape $\boldsymbol{\lambda}$, and is characterized (up to a diagonal change of basis) by the fact that the Jucys-Murphy elements act diagonally by

$$
J_{i}\left(v_{\mathcal{T}}\right)=c(\mathcal{T} \mid i) v_{\mathcal{T}}, \quad i=1, \ldots, n+1
$$

We will not need the explicit formulas for the action of the generators of $H(m, 1, n+1)$ on basis elements $v_{\mathcal{T}}$.

The restriction of irreducible representations of $H(m, 1, n+1)$ to $H(m, 1, n)$ is determined by inclusion of $m$-partitions, that is, for $H(m, 1, n)$-modules, we have

$$
V_{\boldsymbol{\lambda}} \cong \bigoplus_{\boldsymbol{\mu} \subset \boldsymbol{\lambda}, \boldsymbol{\mu} \text { of size } n} V_{\boldsymbol{\mu}}
$$

Moreover, in this decomposition, $V_{\boldsymbol{\mu}}$ is the space spanned by the basis vectors $v_{\mathcal{T}}$, with $\mathcal{T}$ such that the standard $m$-tableau (of size $n$ ) obtained by removing from $\mathcal{T}$ the $m$-node containing $n+1$ is of shape $\boldsymbol{\mu}$.

For a standard $m$-tableau $\mathcal{T}$ of size $n+1$, we denote by $E_{\mathcal{T}}$ the primitive idempotent of $H(m, 1, n+1)$ corresponding to $v_{\mathcal{T}}$, uniquely defined by $E_{\mathcal{T}} v_{\mathcal{T}^{\prime}}=\delta_{\mathcal{T} \mathcal{T}^{\prime}} v_{\mathcal{T}}$. The results recalled above imply that $\left\{E_{\mathcal{T}}\right\}$, where $\mathcal{T}$ runs through the set of standard $m$-tableaux of size $n+1$, is a complete set of pairwise orthogonal primitive idempotents of $H(m, 1, n+1)$. Moreover, we have by construction

$$
J_{i} E_{\mathcal{T}}=E_{\mathcal{T}} J_{i}=c(\mathcal{T} \mid i) E_{\mathcal{T}}, \quad i=1, \ldots, n+1 .
$$

Due to the maximality of the commutative set formed by the Jucys-Murphy elements, the idempotent $E_{\mathcal{T}}$ can be expressed in terms of the elements $J_{i}, i=1, \ldots, n+1$. Let $\gamma$ be the $m$-node of $\mathcal{T}$ containing the number $n+1$. As the $m$-tableau $\mathcal{T}$ is standard, the $m$-node $\gamma$ of $\boldsymbol{\lambda}$ is removable. Let $\mathcal{U}$ be the standard $m$-tableau obtained from $\mathcal{T}$ by removing the $m$-node $\gamma$, and let $\boldsymbol{\mu}$ be the shape of $\mathcal{U}$. By (13) and (14), the inductive formula for $E_{\mathcal{T}}$ in terms of the Jucys-Murphy elements reads

$$
E_{\mathcal{T}}=E_{\mathcal{U}} \prod_{\boldsymbol{\beta}:} \frac{J_{n+1}-c(\boldsymbol{\beta})}{\substack{\boldsymbol{\beta} \neq \boldsymbol{\mathcal { E }}+(\boldsymbol{\mu}) \\ \boldsymbol{\beta} \neq \boldsymbol{\gamma}}}
$$

with the initial condition: $E_{\mathcal{U}_{0}}=1$ for the unique $m$-tableau $\mathcal{U}_{0}$ of size 0 . Here $E_{\mathcal{U}}$ is considered as an element of the algebra $H(m, 1, n+1)$. Note that, due to the restrictions (2)-(4), we have $c(\boldsymbol{\beta}) \neq c(\boldsymbol{\gamma})$ for any $\boldsymbol{\beta} \in \mathcal{E}_{+}(\boldsymbol{\mu})$ such that $\boldsymbol{\beta} \neq \boldsymbol{\gamma}$.

Let $\left\{\mathcal{T}_{1}, \ldots, \mathcal{T}_{a}\right\}$ be the set of pairwise different standard $m$-tableaux which can be obtained from $\mathcal{U}$ by adding an $m$-node with number $n+1$. As a consequence of (13), we have the formula

$$
E_{\mathcal{U}}=\sum_{i=1}^{a} E_{\mathcal{T}_{i}}
$$


The element $J_{n+1}$ satisfies a polynomial equation of finite order so its resolvent is well defined and

$$
E_{\mathcal{U}} \frac{u-c(\mathcal{T} \mid n+1)}{u-J_{n+1}}
$$

is a rational function in an indeterminate $u$ with values in $H(m, 1, n+1)$. Replacing $E_{\mathcal{U}}$ by the right-hand side of (15) and using (14), we obtain that this function is non-singular at $u=c(\mathcal{T} \mid n+1)$ and moreover, due to the restrictions (2)-(4),

$$
\left.E_{\mathcal{U}} \frac{u-c(\mathcal{T} \mid n+1)}{u-J_{n+1}}\right|_{u=c(\mathcal{T} \mid n+1)}=E_{\mathcal{T}}
$$

\section{Fusion formula for the algebra $H(m, 1, n+1)$}

In this section, we prove, in Theorem 1 below, the fusion formula for the primitive idempotents $E_{\mathcal{T}}$. We use the inductive formula (16) for $E_{\mathcal{T}}$.

Let $\phi_{k}$, for $k=1, \ldots, n+1$, be the rational functions in variables $u_{1}, \ldots, u_{k}$ with values in the algebra $H(m, 1, n+1)$ defined by $\phi_{1}\left(u_{1}\right):=\bar{\tau}\left(u_{1}\right)$ and, for $k=1, \ldots, n$,

$$
\begin{aligned}
& \phi_{k+1}\left(u_{1}, \ldots, u_{k}, u_{k+1}\right):=\bar{\sigma}_{k}\left(u_{k+1}, u_{k}\right) \phi_{k}\left(u_{1}, \ldots, u_{k-1}, u_{k+1}\right) \sigma_{k}^{-1} \\
& \quad=\bar{\sigma}_{k}\left(u_{k+1}, u_{k}\right) \bar{\sigma}_{k-1}\left(u_{k+1}, u_{k-1}\right) \ldots \bar{\sigma}_{1}\left(u_{k+1}, u_{1}\right) \bar{\tau}\left(u_{k+1}\right) \sigma_{1}^{-1} \ldots \sigma_{k-1}^{-1} \sigma_{k}^{-1} .
\end{aligned}
$$

Define the following rational function $\Phi$ in variables $u_{1}, \ldots, u_{n+1}$ with values in $H(m, 1, n+1)$ :

$$
\Phi\left(u_{1}, \ldots, u_{n+1}\right):=\phi_{n+1}\left(u_{1}, \ldots, u_{n}, u_{n+1}\right) \phi_{n}\left(u_{1}, \ldots, u_{n-1}, u_{n}\right) \cdots \phi_{1}\left(u_{1}\right) .
$$

Let $\boldsymbol{\lambda}$ be an $m$-partition of size $n+1$ and $\mathcal{T}$ a standard $m$-tableau of shape $\boldsymbol{\lambda}$. For $i=$ $1, \ldots, n+1$, we set $c_{i}:=c(\mathcal{T} \mid i)$.

Theorem 1. The idempotent $E_{\mathcal{T}}$ corresponding to the standard $m$-tableau $\mathcal{T}$ of shape $\boldsymbol{\lambda}$ can be obtained by the following consecutive evaluations

$$
E_{\mathcal{T}}=\left.\left.\left.\mathrm{F}_{\lambda} \Phi\left(u_{1}, \ldots, u_{n+1}\right)\right|_{u_{1}=c_{1}} \cdots\right|_{u_{n}=c_{n}}\right|_{u_{n+1}=c_{n+1}},
$$

with $\mathrm{F}_{\boldsymbol{\lambda}}$ defined in (12).

We will prove the theorem in this section in several steps.

Until the end of the text, $\boldsymbol{\gamma}$ and $\boldsymbol{\delta}$ denote the $m$-nodes of $\mathcal{T}$ containing the numbers $n+1$ and $n$ respectively; $\mathcal{U}$ is the standard $m$-tableau obtained from $\mathcal{T}$ by removing $\gamma$, and $\boldsymbol{\mu}$ is the shape of $\mathcal{U}$; also, $\mathcal{W}$ is the standard $m$-tableau obtained from $\mathcal{U}$ by removing the $m$-node $\boldsymbol{\delta}$ and $\boldsymbol{\nu}$ is the shape of $\mathcal{W}$.

For a standard $m$-tableau $\mathcal{V}$ of size $N$, we define the following rational function in a variable $u$ with complex values

$$
F_{\mathcal{V}}(u):=\frac{u-c(\mathcal{V} \mid N)}{\left(u-v_{1}\right) \cdots\left(u-v_{m}\right)} \prod_{i=1}^{N-1} \frac{(u-c(\mathcal{V} \mid i))^{2}}{\left(u-q^{2} c(\mathcal{V} \mid i)\right)\left(u-q^{-2} c(\mathcal{V} \mid i)\right)} ;
$$

by convention, $F_{\mathcal{V}}(u):=\frac{u-c(\mathcal{V} \mid 1)}{\left(u-v_{1}\right) \cdots\left(u-v_{m}\right)}$ for $N=1$.

Proposition 2. We have

$$
F_{\mathcal{T}}(u) \phi_{n+1}\left(c_{1}, \ldots, c_{n}, u\right) E_{\mathcal{U}}=\frac{u-c_{n+1}}{u-J_{n+1}} E_{\mathcal{U}} .
$$


Proof. We prove (18) by induction on $n$. As $J_{1}=\tau$, we have by (7)

$$
\frac{u-c_{1}}{u-J_{1}}=\frac{u-c_{1}}{\left(u-v_{1}\right) \cdots\left(u-v_{m}\right)} \bar{\tau}(u),
$$

which verifies the basis of induction $(n=0)$.

We have: $E_{\mathcal{W}} E_{\mathcal{U}}=E_{\mathcal{U}}$ and $E_{\mathcal{W}}$ commutes with $\sigma_{n}$. Rewrite the left-hand side of (18) as

$$
F_{\mathcal{T}}(u) \bar{\sigma}_{n}\left(u, c_{n}\right) \cdot \phi_{n}\left(c_{1}, \ldots, c_{n-1}, u\right) E_{\mathcal{W}} \cdot \sigma_{n}^{-1} E_{\mathcal{U}}
$$

By the induction hypothesis we have for the left-hand side of (18)

$$
F_{\mathcal{T}}(u)\left(F_{\mathcal{U}}(u)\right)^{-1} \bar{\sigma}_{n}\left(u, c_{n}\right) \frac{u-c_{n}}{u-J_{n}} \sigma_{n}^{-1} E_{\mathcal{U}}
$$

Since $J_{n+1}$ commutes with $E_{\mathcal{U}}$, the equality (18) is equivalent to

$$
\begin{aligned}
& F_{\mathcal{T}}(u)\left(F_{\mathcal{U}}(u)\right)^{-1}\left(u-c_{n}\right) \sigma_{n}^{-1}\left(u-J_{n+1}\right) E_{\mathcal{U}} \\
& =\frac{\left(u-c_{n+1}\right)\left(u-c_{n}\right)^{2}}{\left(u-q^{2} c_{n}\right)\left(u-q^{-2} c_{n}\right)}\left(u-J_{n}\right) \bar{\sigma}_{n}\left(c_{n}, u\right) E_{\mathcal{U}}
\end{aligned}
$$

(the inverse of $\bar{\sigma}_{n}\left(u, c_{n}\right)$ is calculated with the help of (6)). By (17),

$$
F_{\mathcal{T}}(u)\left(F_{\mathcal{U}}(u)\right)^{-1}\left(u-c_{n}\right)=\left(u-c_{n+1}\right) \frac{\left(u-c_{n}\right)^{2}}{\left(u-q^{2} c_{n}\right)\left(u-q^{-2} c_{n}\right)} .
$$

Therefore, to prove (19), it remains to show that

$$
\sigma_{n}^{-1}\left(u-J_{n+1}\right) E_{\mathcal{U}}=\left(u-J_{n}\right) \bar{\sigma}_{n}\left(c_{n}, u\right) E_{\mathcal{U}} .
$$

Replacing $J_{n+1}$ by $\sigma_{n} J_{n} \sigma_{n}$, we write the left-hand side of (20) in the form

$$
\left(u \sigma_{n}^{-1}-J_{n} \sigma_{n}\right) E_{\mathcal{U}}
$$

As $J_{n} E_{\mathcal{U}}=c_{n} E_{\mathcal{U}}$, the right-hand side of (20) is

$$
\left(u \sigma_{n}-J_{n} \sigma_{n}+\left(q-q^{-1}\right)\left(u-c_{n}\right) \frac{u}{c_{n}-u}\right) E_{\mathcal{U}}
$$

and thus coincides with (21).

To prove Theorem 1, we need the following information about the behavior of the rational function $F_{\mathcal{T}}(u)$ at $u=c_{n+1}$.

Proposition 3. The rational function $F_{\mathcal{T}}(u)$ is non-singular at $u=c_{n+1}$, and moreover

$$
F_{\mathcal{T}}\left(c_{n+1}\right)=\mathrm{F}_{\boldsymbol{\lambda}} \mathrm{F}_{\boldsymbol{\mu}}^{-1}
$$

We will prove this proposition with the help of Lemmas 4 and 5 below, which involve the combinatorics of multi-partitions.

Lemma 4. We have

$$
F_{\mathcal{T}}(u)=\left(u-c_{n+1}\right) \prod_{\boldsymbol{\beta} \in \mathcal{E}_{-}(\boldsymbol{\mu})}(u-c(\boldsymbol{\beta})) \prod_{\boldsymbol{\alpha} \in \mathcal{E}_{+}(\boldsymbol{\mu})}(u-c(\boldsymbol{\alpha}))^{-1} .
$$


Proof. The proof is by induction on $n$. For $n=0$, we have

$$
F_{\mathcal{T}}(u)=\frac{u-c_{1}}{\left(u-v_{1}\right) \cdots\left(u-v_{m}\right)},
$$

which is equal to the right-hand side of (22).

Now, for $n>0$, we rewrite (17) for $\mathcal{V}=\mathcal{T}$ as

$$
F_{\mathcal{T}}(u)=\frac{u-c_{n+1}}{\left(u-v_{1}\right) \cdots\left(u-v_{m}\right)} \frac{\left(u-c_{n}\right)^{2}}{\left(u-q^{2} c_{n}\right)\left(u-q^{-2} c_{n}\right)} \prod_{i=1}^{n-1} \frac{\left(u-c_{i}\right)^{2}}{\left(u-q^{2} c_{i}\right)\left(u-q^{-2} c_{i}\right)} .
$$

Using the induction hypothesis, we obtain

$$
F_{\mathcal{T}}(u)=\frac{\left(u-c_{n+1}\right)\left(u-c_{n}\right)^{2}}{\left(u-q^{2} c_{n}\right)\left(u-q^{-2} c_{n}\right)} \prod_{\boldsymbol{\beta} \in \mathcal{E}_{-}(\boldsymbol{\nu})}(u-c(\boldsymbol{\beta})) \prod_{\boldsymbol{\alpha} \in \mathcal{E}_{+}(\boldsymbol{\nu})}(u-c(\boldsymbol{\alpha}))^{-1} .
$$

Denote by $\boldsymbol{\delta}_{t}$ and $\boldsymbol{\delta}_{b}$ the $m$-nodes which are, respectively, just above and just below $\boldsymbol{\delta}, \boldsymbol{\delta}_{l}$ and $\boldsymbol{\delta}_{r}$ the $m$-nodes which are, respectively, just on the left and just on the right of $\boldsymbol{\delta}$; it might happen that one of the coordinates of $\boldsymbol{\delta}_{t}$ (or $\boldsymbol{\delta}_{l}$ ) is not positive, and in this situation, by definition, $\boldsymbol{\delta}_{t} \notin \mathcal{E}_{-}(\boldsymbol{\nu})$ (or $\boldsymbol{\delta}_{l} \notin \mathcal{E}_{-}(\boldsymbol{\nu})$ ). It is straightforward to see that:

- If $\boldsymbol{\delta}_{t}, \boldsymbol{\delta}_{l} \notin \mathcal{E}_{-}(\boldsymbol{\nu})$ then

$$
\mathcal{E}_{-}(\boldsymbol{\mu})=\mathcal{E}_{-}(\boldsymbol{\nu}) \cup\{\boldsymbol{\delta}\} \quad \text { and } \quad \mathcal{E}_{+}(\boldsymbol{\mu})=\left(\mathcal{E}_{+}(\boldsymbol{\nu}) \cup\left\{\boldsymbol{\delta}_{b}, \boldsymbol{\delta}_{r}\right\}\right) \backslash\{\boldsymbol{\delta}\}
$$

- If $\boldsymbol{\delta}_{t} \in \mathcal{E}_{-}(\boldsymbol{\nu})$ and $\boldsymbol{\delta}_{l} \notin \mathcal{E}_{-}(\boldsymbol{\nu})$ then

$$
\mathcal{E}_{-}(\boldsymbol{\mu})=\left(\mathcal{E}_{-}(\boldsymbol{\nu}) \cup\{\boldsymbol{\delta}\}\right) \backslash\left\{\boldsymbol{\delta}_{t}\right\} \quad \text { and } \quad \mathcal{E}_{+}(\boldsymbol{\mu})=\left(\mathcal{E}_{+}(\boldsymbol{\nu}) \cup\left\{\boldsymbol{\delta}_{b}\right\}\right) \backslash\{\boldsymbol{\delta}\} .
$$

- If $\boldsymbol{\delta}_{t} \notin \mathcal{E}_{-}(\boldsymbol{\nu})$ and $\boldsymbol{\delta}_{l} \in \mathcal{E}_{-}(\boldsymbol{\nu})$ then

$$
\mathcal{E}_{-}(\boldsymbol{\mu})=\left(\mathcal{E}_{-}(\boldsymbol{\nu}) \cup\{\boldsymbol{\delta}\}\right) \backslash\left\{\boldsymbol{\delta}_{l}\right\} \quad \text { and } \quad \mathcal{E}_{+}(\boldsymbol{\mu})=\left(\mathcal{E}_{+}(\boldsymbol{\nu}) \cup\left\{\boldsymbol{\delta}_{r}\right\}\right) \backslash\{\boldsymbol{\delta}\} .
$$

- If $\boldsymbol{\delta}_{t}, \boldsymbol{\delta}_{l} \in \mathcal{E}_{-}(\boldsymbol{\nu})$ then

$$
\mathcal{E}_{-}(\boldsymbol{\mu})=\left(\mathcal{E}_{-}(\boldsymbol{\nu}) \cup\{\boldsymbol{\delta}\}\right) \backslash\left\{\boldsymbol{\delta}_{t}, \boldsymbol{\delta}_{l}\right\} \quad \text { and } \quad \mathcal{E}_{+}(\boldsymbol{\mu})=\mathcal{E}_{+}(\boldsymbol{\nu}) \backslash\{\boldsymbol{\delta}\} .
$$

In each case, using that $c\left(\boldsymbol{\delta}_{t}\right)=c\left(\boldsymbol{\delta}_{r}\right)=q^{2} c_{n}$ and $c\left(\boldsymbol{\delta}_{b}\right)=c\left(\boldsymbol{\delta}_{l}\right)=q^{-2} c_{n}$, it follows that the right-hand side of (23) is equal to

$$
\left(u-c_{n+1}\right) \prod_{\boldsymbol{\beta} \in \mathcal{E}_{-}(\boldsymbol{\mu})}(u-c(\boldsymbol{\beta})) \prod_{\boldsymbol{\alpha} \in \mathcal{E}_{+}(\boldsymbol{\mu})}(u-c(\boldsymbol{\alpha}))^{-1},
$$

which establishes the formula (22).

Lemma 5. We have

$$
\prod_{\boldsymbol{\beta} \in \mathcal{E}_{-}(\boldsymbol{\mu})}\left(c_{n+1}-c(\boldsymbol{\beta})\right) \prod_{\boldsymbol{\alpha} \in \mathcal{E}_{+}(\boldsymbol{\mu}) \backslash\{\gamma\}}\left(c_{n+1}-c(\boldsymbol{\alpha})\right)^{-1}=\mathrm{F}_{\lambda} \mathrm{F}_{\boldsymbol{\mu}}^{-1} .
$$


Proof. 1. The definition (12), for a partition $\lambda$, reduces to

$$
\mathrm{F}_{\lambda}:=\prod_{\alpha \in \lambda} \frac{q^{c c(\alpha)}}{\left[h_{\lambda}(\alpha)\right]_{q}}
$$

The Lemma 5 for a partition $\lambda$ is established in [8, Lemma 3.2].

2. Set $k=\operatorname{pos}(\gamma)$. Define, for an $m$-partition $\boldsymbol{\theta}$,

$$
\widetilde{\mathrm{F}}_{\boldsymbol{\theta}}:=\prod_{\boldsymbol{\alpha} \in \boldsymbol{\theta}} \frac{q^{c c(\boldsymbol{\alpha})}}{\left[h_{\boldsymbol{\theta}}(\boldsymbol{\alpha})\right]_{q}},
$$

and, for $j=1, \ldots, m$ such that $j \neq k$,

$$
\mathrm{F}_{\boldsymbol{\theta}}^{(j)}:=\prod_{\substack{\boldsymbol{\alpha} \in \boldsymbol{\theta} \\ \operatorname{pos}(\boldsymbol{\alpha})=k}} \frac{q^{-c c(\boldsymbol{\alpha})}}{v_{k} q^{-h_{\boldsymbol{\theta}}^{(j)}(\boldsymbol{\alpha})}-v_{j} q^{h_{\boldsymbol{\theta}}^{(j)}(\boldsymbol{\alpha})}} \prod_{\substack{\boldsymbol{\alpha} \in \boldsymbol{\theta} \\ \operatorname{pos}(\boldsymbol{\alpha})=j}} \frac{q^{-c c(\boldsymbol{\alpha})}}{v_{j} q^{-h_{\boldsymbol{\theta}}^{(k)}(\boldsymbol{\alpha})}-v_{k} q^{h_{\boldsymbol{\theta}}^{(k)}(\boldsymbol{\alpha})}} .
$$

By (12), we have

$$
\mathrm{F}_{\boldsymbol{\theta}}=\widetilde{\mathrm{F}}_{\boldsymbol{\theta}} \prod_{\substack{j=1, \ldots, m \\ j \neq k}} \mathrm{~F}_{\boldsymbol{\theta}}^{(j)}
$$

Fix $j \in\{1, \ldots, m\}$ such that $j \neq k$. We shall show that

$$
\prod_{\substack{\boldsymbol{\beta} \in \mathcal{E}_{-}(\boldsymbol{\mu}) \\ \operatorname{pos}(\boldsymbol{\beta})=j}}\left(c_{n+1}-c(\boldsymbol{\beta})\right) \prod_{\substack{\boldsymbol{\alpha} \in \mathcal{E}_{+}(\boldsymbol{\mu}) \backslash\{\boldsymbol{\gamma}\} \\ \operatorname{pos}(\boldsymbol{\alpha})=j}}\left(c_{n+1}-c(\boldsymbol{\alpha})\right)^{-1}=\mathrm{F}_{\boldsymbol{\lambda}}^{(j)}\left(\mathrm{F}_{\boldsymbol{\mu}}^{(j)}\right)^{-1} .
$$

Let $p_{1}<p_{2}<\cdots<p_{s}$ be positive integers such that the $j$-th partition of $\boldsymbol{\mu}$ is $\left(\mu_{1}, \ldots, \mu_{p_{s}}\right)$ with

$$
\mu_{1}=\cdots=\mu_{p_{1}}>\mu_{p_{1}+1}=\cdots=\mu_{p_{2}}>\cdots>\mu_{p_{s-1}+1}=\cdots=\mu_{p_{s}}>0 .
$$

We set $p_{0}:=0, p_{s+1}:=+\infty$ and $\mu_{p_{s+1}}:=0$. Assume that the $m$-node $\gamma$ lies in the line $x$ and column $y$. The left-hand side of (26) is equal to

$$
\prod_{b=1}^{s}\left(v_{k} q^{2(y-x)}-v_{j} q^{2\left(\mu_{p_{b}}-p_{b}\right)}\right) \prod_{b=1}^{s+1}\left(v_{k} q^{2(y-x)}-v_{j} q^{2\left(\mu_{p_{b}}-p_{b-1}\right)}\right)^{-1} .
$$

The factors in the product (24) correspond to $m$-nodes of an $m$-partition. The $m$-nodes lying neither in the column $y$ of the $k$-th diagrams (of $\boldsymbol{\lambda}$ or $\boldsymbol{\mu}$ ) nor in the line $x$ of the $j$-th diagrams do not contribute to the right-hand side of (26). Let $t \in\{0, \ldots, s\}$ be such that $p_{t}<x \leq p_{t+1}$. The contribution from the $m$-nodes in the column $y$ and lines $1, \ldots, p_{t}$ of the $k$-th diagrams is

$$
\prod_{b=1}^{t}\left(\prod_{a=p_{b-1}+1}^{p_{b}} \frac{v_{k} q^{-\left(\mu_{p_{b}}-y+x-a\right)}-v_{j} q^{\left(\mu_{p_{b}}-y+x-a\right)}}{v_{k} q^{-\left(\mu_{p_{b}}-y+x-a+1\right)}-v_{j} q^{\left(\mu_{p_{b}}-y+x-a+1\right)}}\right)
$$

the contribution from the $m$-nodes in the column $y$ and lines $p_{t}+1, \ldots, x$ of the $k$-th diagrams is

$$
\prod_{a=p_{t}+1}^{x-1}\left(\frac{v_{k} q^{-\left(\mu_{p_{t+1}}-y+x-a\right)}-v_{j} q^{\left(\mu_{p_{t+1}}-y+x-a\right)}}{v_{k} q^{-\left(\mu_{p_{t+1}}-y+x-a+1\right)}-v_{j} q^{\left(\mu_{p_{t+1}}-y+x-a+1\right)}}\right) \frac{q^{-c c(\gamma)}}{v_{k} q^{-\left(\mu_{p_{t+1}}-y+1\right)}-v_{j} q^{\left(\mu_{p_{t+1}}-y+1\right)}} .
$$


The contribution from the $m$-nodes lying in the line $x$ of the $j$-th diagrams is

$$
\prod_{b=t+1}^{s} \prod_{a=\mu_{p_{b+1}}+1}^{\mu_{p_{b}}} \frac{v_{j} q^{-\left(y-a+p_{b}-x\right)}-v_{k} q^{\left(y-a+p_{b}-x\right)}}{v_{j} q^{-\left(y-a+p_{b}-x+1\right)}-v_{k} q^{\left(y-a+p_{b}-x+1\right)}} .
$$

After straightforward simplifications, we obtain for the right-hand side of (26)

$$
\begin{aligned}
q^{x-y} & \prod_{b=1}^{s}\left(v_{k} q^{-\left(\mu_{p_{b}}-y+x-p_{b}\right)}-v_{j} q^{\left(\mu_{p_{b}}-y+x-p_{b}\right)}\right) \\
& \times \prod_{b=1}^{s+1}\left(v_{k} q^{-\left(\mu_{p_{b}}-y+x-p_{b-1}\right)}-v_{j} q^{\left(\mu_{p_{b}}-y+x-p_{b-1}\right)}\right)^{-1} .
\end{aligned}
$$

The comparison of (27) and (28) concludes the proof of the formula (26).

3. The assertion of the lemma is a consequence of the formulas (25), (26) together with the part 1 of the proof.

Proof of the Proposition 3. The formula (22) shows that the rational function $F_{\mathcal{T}}(u)$ is nonsingular at $u=c_{n+1}$, and moreover

$$
F_{\mathcal{T}}\left(c_{n+1}\right)=\prod_{\boldsymbol{\beta} \in \mathcal{E}_{-}(\boldsymbol{\mu})}\left(c_{n+1}-c(\boldsymbol{\beta})\right) \prod_{\boldsymbol{\alpha} \in \mathcal{E}_{+}(\boldsymbol{\mu}) \backslash\{\gamma\}}\left(c_{n+1}-c(\boldsymbol{\alpha})\right)^{-1} .
$$

We use the Lemma 5 to conclude the proof of the proposition.

Proof of Theorem 1. The theorem follows, by induction on $n$, from the formula (16) together with Propositions 2 and 3.

Example. Consider, for $m=2$, the standard 2-tableau $\left(\begin{array}{ll}1 & 3\end{array}, 2,2\right)$. The idempotent of the algebra $H(2,1,3)$ corresponding to this standard 2-tableau reads, by the Theorem 1 ,

$$
\frac{\bar{\sigma}_{2}\left(v_{1} q^{2}, v_{2}\right) \bar{\sigma}_{1}\left(v_{1} q^{2}, v_{1}\right) \bar{\tau}\left(v_{1} q^{2}\right) \sigma_{1}^{-1} \sigma_{2}^{-1} \bar{\sigma}_{1}\left(v_{2}, v_{1}\right) \bar{\tau}\left(v_{2}\right) \sigma_{1}^{-1} \bar{\tau}\left(v_{1}\right)}{\left(q+q^{-1}\right)\left(v_{1} q^{-1}-v_{2} q\right)\left(v_{1}-v_{2}\right)\left(v_{2} q^{-2}-v_{1} q^{2}\right)}
$$

\section{Remarks on the classical limit}

Recall that the group ring $\mathbb{C} G(m, 1, n+1)$ of the complex reflection group $G(m, 1, n+1)$ is obtained by taking the classical limit: $q \mapsto \pm 1$ and $v_{i} \mapsto \xi_{i}, i=1, \ldots, m$, where $\left\{\xi_{1}, \ldots, \xi_{m}\right\}$ is the set of distinct $m$-th roots of unity. The "classical limit" of the generators $\tau, \sigma_{1}, \ldots, \sigma_{n}$ of $H(m, 1, n+1)$ we denote by $t, s_{1}, \ldots, s_{n}$.

1. Consider the Baxterized elements (5) with spectral parameters of the form $v_{p} q^{2 a}$ and $v_{p^{\prime}} q^{2 a^{\prime}}$ with $p, p^{\prime} \in\{1, \ldots, m\}$. One directly finds that

$$
\lim _{q \rightarrow 1} \lim _{v_{i} \rightarrow \xi_{i}} \bar{\sigma}_{i}\left(v_{p} q^{2 a}, v_{p^{\prime}} q^{2 a^{\prime}}\right)=s_{i}+\frac{\delta_{p, p^{\prime}}}{a-a^{\prime}} .
$$

For the Artin generators $\tilde{s}_{1}, \ldots, \tilde{s}_{n}$ of the symmetric group $S_{n+1}$, the standard Baxterized elements are given by the rational functions

$$
\tilde{s}_{i}+\frac{1}{a-a^{\prime}} \quad \text { for } i=1, \ldots, n
$$


In view of (29), we define generalized Baxterized elements for the group $G(m, 1, n+1)$ as the following functions

$$
\bar{s}_{i}\left(p, p^{\prime}, a, a^{\prime}\right):=s_{i}+\frac{\delta_{p, p^{\prime}}}{a-a^{\prime}} \quad \text { for } \quad i=1, \ldots, n .
$$

These elements satisfy the following Yang-Baxter equation with spectral parameters

$$
\begin{aligned}
& \bar{s}_{i}\left(p, p^{\prime}, a, a^{\prime}\right) \bar{s}_{i+1}\left(p, p^{\prime \prime}, a, a^{\prime \prime}\right) \bar{s}_{i}\left(p^{\prime}, p^{\prime \prime}, a^{\prime}, a^{\prime \prime}\right) \\
& =\bar{s}_{i+1}\left(p^{\prime}, p^{\prime \prime}, a^{\prime}, a^{\prime \prime}\right) \bar{s}_{i}\left(p, p^{\prime \prime}, a, a^{\prime \prime}\right) \bar{s}_{i+1}\left(p, p^{\prime}, a, a^{\prime}\right) .
\end{aligned}
$$

The Baxterized elements (30) have been used in [14] for a fusion procedure for the complex reflection group $G(m, 1, n+1)$.

2. It is immediate that

$$
\lim _{v_{i} \rightarrow \xi_{i}} \mathfrak{a}_{0}(r)=r^{m}-1 \quad \text { and } \quad \lim _{v_{i} \rightarrow \xi_{i}} \mathfrak{a}_{i}(r)=r^{m-i} \quad \text { for } \quad i=1, \ldots, m,
$$

where $\mathfrak{a}_{i}(r), i=0, \ldots, m$, are defined in (8). It follows from (10) that

$$
\lim _{v_{i} \rightarrow \xi_{i}} \bar{\tau}(r)=\sum_{i=0}^{m-1} r^{m-1-i} t^{i}
$$

The rational function $\bar{t}$ defined by $\bar{t}(r):=\frac{1}{m} \sum_{i=0}^{m-1} r^{m-i} t^{i}$ with values in $\mathbb{C} G(m, 1, n+1)$ was used in [14] for a fusion procedure for the complex reflection group $G(m, 1, n+1)$.

3. Define, for an $m$-partition $\boldsymbol{\lambda}$,

$$
f_{\boldsymbol{\lambda}}:=\left(\prod_{\boldsymbol{\alpha} \in \boldsymbol{\lambda}} h_{\boldsymbol{\lambda}}(\boldsymbol{\alpha})\right)^{-1} .
$$

The classical limit of $F_{\boldsymbol{\lambda}}$ is proportional to $f_{\boldsymbol{\lambda}}$. More precisely, we have

$$
\lim _{q \rightarrow 1} \lim _{v_{i} \rightarrow \xi_{i}} \mathrm{~F}_{\boldsymbol{\lambda}}=\mathfrak{x}_{\boldsymbol{\lambda}} f_{\boldsymbol{\lambda}}, \quad \text { where } \mathfrak{x}_{\boldsymbol{\lambda}}=\frac{1}{m^{n}} \prod_{\boldsymbol{\alpha} \in \boldsymbol{\lambda}} \xi_{\operatorname{pos}(\boldsymbol{\alpha})} .
$$

The formula (32) is obtained directly from (12) since

$$
\prod_{\substack{i=1 \\ i \neq k}}^{m}\left(\xi_{k}-\xi_{i}\right)=m / \xi_{k} \quad \text { for } k=1, \ldots, m
$$

4. Using formulas (29), (31) and (32), it is straightforward to check that the classical limit of the fusion procedure for $H(m, 1, n+1)$ given by the Theorem 1 leads to the fusion procedure [14] for the group $G(m, 1, n+1)$. Also, for $m=1$, Theorem 1 coincides with the fusion procedure [8] for the Hecke algebra and, in the classical limit, with the fusion procedure [12] for the symmetric group.

\section{Acknowledgements}

We thank the anonymous referees for valuable suggestions. 


\section{References}

[1] Ariki S., On the semi-simplicity of the Hecke algebra of $(\mathbb{Z} / r \mathbb{Z})$ 乙 $\mathfrak{S}_{n}$, J. Algebra 169 (1994), 216-225.

[2] Ariki S., Koike K., A Hecke algebra of $(\mathbb{Z} / r \mathbb{Z}) \imath \mathfrak{S}_{n}$ and construction of its irreducible representations, Adv. Math. 106 (1994), 216-243.

[3] Broué M., Malle G., Zyklotomische Heckealgebren. Représentations unipotentes génériques et blocs des groupes réductifs finis, Astérisque 212 (1993), 119-189.

[4] Cherednik I.V., A new interpretation of Gel'fand-Tzetlin bases, Duke Math. J. 54 (1987), 563-577.

[5] Chlouveraki M., Jacon N., Schur elements for the Ariki-Koike algebra and applications, J. Algebraic Combin. 35 (2012), 291-311, arXiv:1105.5910.

[6] Geck M., Iancu L., Malle G., Weights of Markov traces and generic degrees, Indag. Math. (N.S.) 11 (2000), 379-397.

[7] Isaev A.P., Molev A.I., Ogievetsky O.V., Idempotents for Birman-Murakami-Wenzl algebras and reflection equation, arXiv:1111.2502.

[8] Isaev A.P., Molev A.I., Os'kin A.F., On the idempotents of Hecke algebras, Lett. Math. Phys. 85 (2008), 79-90, arXiv:0804.4214.

[9] Isaev A.P., Ogievetsky O.V., On Baxterized solutions of reflection equation and integrable chain models, Nuclear Phys. B $\mathbf{7 6 0}$ (2007), 167-183, math-ph/0510078.

[10] Kulish P.P., Yang-Baxter equation and reflection equations in integrable models, in Low-Dimensional Models in Statistical Physics and Quantum Field Theory (Schladming, 1995), Lecture Notes in Phys., Vol. 469, Springer, Berlin, 1996, 125-144.

[11] Mathas A., Matrix units and generic degrees for the Ariki-Koike algebras, J. Algebra 281 (2004), 695-730, math.RT/0108164.

[12] Molev A.I., On the fusion procedure for the symmetric group, Rep. Math. Phys. 61 (2008), 181-188, math.RT/0612207.

[13] Ogievetsky O.V., Poulain d'Andecy L., On representations of cyclotomic Hecke algebras, Modern Phys. Lett. A 26 (2011), 795-803, arXiv:1012.5844.

[14] Ogievetsky O.V., Poulain d'Andecy L., Fusion formula for Coxeter groups of type B and complex reflection groups $G(m, 1, n)$, arXiv:1111.6293.

[15] Ogievetsky O.V., Poulain d'Andecy L., Induced representations and traces for chains of affine and cyclotomic Hecke algebras, arXiv:1312.6980. 